\title{
Influence of Organ Motion and Contrast Enhancement on Image Registration
}

\author{
A. Melbourne, D. Atkinson, and D. Hawkes \\ Centre for Medical Image Computing, University College London
}

\begin{abstract}
We present an exploration of the interplay between the extent of organ motion and contrast-enhancement on image registration success. A model of a DCE MRI of the liver incorporating an isotropic elastic non-rigid deformation is used to simulate both breathing and breath-hold data, a volume-preserving modification for tumour regions is included. Contrast enhancement is simulated by applying a pharmacokinetic model. For each simulated dataset, a direct fluid registration of each image to the first in the dataset is compared to a contrast-enhancement guided method known as Progressive Principal Component Registration (PPCR). Analysis of the correction to the deformation fields, tumour volume change and dispersion of joint image histograms are used to show the importance of motion type on PPCR success and of enhancement level on direct fluid registration success. For breathing motion, PPCR registers groups of images to separate locations, but maintains enhancing tumour volume. This is not the case for direct registration with volume changes of up to $7 \%$. For inconsistent breath-hold depth, PPCR out-performs direct registration, particularly for large enhancement levels. Analysis of the joint histograms suggests that the generation of target images using PPCR reduces dispersion due to contrast enhancement. Since this distinction is not made using direct registration, it is unable to register images when large enhancement intensity changes are present.
\end{abstract}

\section{Introduction}

Dynamic Contrast-Enhanced Magnetic Resonance Imaging (DCE-MRI) is often used in diagnostic imaging. An imaging signal that relates to the biological uptake of contrast agent is observed at many time points and dynamic information is acquired. The process of contrast enhancement can be modelled using a suitable physiological description of enhancement processes ([1, [2]).

A DCE-MRI scan often takes minutes, therefore the patient must breath and organs of interest such as the liver will move over time. Image registration can be used to re-align organs within the images, allowing further analysis for use in diagnosis and therapy evaluation. However, registration methods require that images being registered have the same information and structure, but this requirement is not met in DCE-MRI, since the enhancement introduces new information into the images. Recently, solutions that use the enhancement information to guide the registration have been proposed ([3], 4]). The latter 
solution makes assumptions about the nature of organ motion and enhancement. We have therefore developed a simulated deformation model of a breathing liver in an individual who either free breathing or repeating breath-holds in order to evaluate registration success when varying motion and enhancement parameters in DCE-MRI. We use this method to better understand the conditions of success for two registration methods, fluid based registration and PPCR, and to better understand where they fail and to gain insight on the reasons for this failure. This will enable us to design better algorithms for these applications.

\section{Methods}

\subsection{A Liver Model}

We seek to generate a deformation model of the liver that will allow it to appear to deform realistically and reversibly. Breathing motion is cyclic and undergoes a hysteretic motion, although the cycle may not be closed and its end point may drift over time [5]. Approximately $70 \%$ of motion is in the superior-inferior direction, with motion of a smaller extent in both the anterior-posterior $(24 \%)$ and medial-lateral $(7 \%)$ directions 6 . For this reason, results are shown for 2D motion, neglecting small medial-lateral deformations. To ensure a model that is both general and that has good deformation properties, we model a global image deformation, in which organ specific motion is induced by careful placement of forces. Deforming forces are placed in order to mimic breathing motion; they are strongest in the region of the lungs and weighted toward a deformation in the superior-inferior direction. We require our forces to be time-varying, allowing the generation of a cyclic model meeting the properties discussed above.

The forces in each direction can be described as Gaussian point forces. A location is chosen at a point $\left[x_{0}, y_{0}\right]$ and a force applied symmetrically around this point that has time-varying magnitude $A(t)$, and an extent $b$ (Equation 1).

$$
\mathbf{F}(x, y, t)=\frac{A(t)}{b \sqrt{2 \pi}} \exp \frac{-\left(\left(x_{0}-x\right)^{2}+\left(y_{0}-y\right)^{2}\right)}{2 b^{2}}
$$

The breathing model applies two forces of the above style, both located in the lung region: a large superior-inferior force, modulated in magnitude with a sinewave $(A(t)=\sin (t))$ and a smaller anterior-posterior force modulated with a cosine. This results in elliptical motion, which is greatest in magnitude in the diaphragm region of the image. Given this force-field, the resultant displacement field is found by the solution of the isotropic linear elastic equation.

We now add a contrast enhancement model; for this we require a segmentation. We segment four regions (and background) representing, the bulk liver, tumours, the vascular tumour boundary and major blood vessels. The enhancement process in each of these can be modelled according to observed physical properties. Vascular features including blood vessels and tumour boundary angiogenesis enhance rapidly. Enhancement also fades rapidly from these regions. 
A physiological description of this process is described by the Kety model (Equation 22) for total contrast agent concentration with time $C_{T}(t)$ 2]. The parameters $K^{\text {trans }}, v_{p}, v_{e}$ correspond to the volume transfer coefficient of contrast agent between blood plasma and extracellular-extravascular space (EES), and the fractional volumes of blood plasma and EES, respectively. $C_{p}(t)$ is the 'Arterial Input Function' describing the temporal injection of contrast agent into the region of interest by the arteries.

$$
\mathbf{C}_{T}(\mathbf{t})=v_{p} \mathbf{C}_{p}(\mathbf{t})+K^{\text {trans }} \int_{0}^{t} \mathbf{C}_{p}\left(\mathbf{t}^{\prime}\right) \exp \left[\frac{-K^{\text {trans }}}{v_{e}}\left(\mathbf{t}-\mathbf{t}^{\prime}\right)\right] d t^{\prime}
$$

A correct Arterial Input Function (AIF) is often difficult to determine, so an empirical model may be used or may be determined from the data. In addition, the liver has a dual blood supply (25\% from the aorta and $75 \%$ from the gastrointestinal system) which must also be modelled. Here we use an empirical model from [7, a dual blood supply model will be included as further work. We alter the three parameters $K^{T}, v_{p}, v_{e}$ to give enhancement profiles with the behaviour we wish each region to display.

Our deformation model is intrinsically isotropic, with linear deformation properties, a situation which is implausible. In the breast, tumour tissue is found to be up to 15 times stiffer than normal breast tissue 88. For this reason, we seek to modify our deformation fields retrospectively to ensure that tumours move rigidly. We segment the tumours and give every pixel in the tumour the mean deformation of the original values. To ensure smoothness at the boundary, we use a Gaussian filter to smooth the deformations together.

\subsection{Image Registration by PPCR}

The Progressive Principal Component Registration (PPCR) method used in [4] uses a Principal Components Analysis (PCA) to represent DCE-MRI data in the time domain. Each pixel in the image, $\mathbf{A}_{i j}$, as a function of time, is represented by a linear combination of the principal component eigenvectors (Equation 3 with equality for $\eta=M$, the total number of principal components, which is also equal to the number of time-points). Common pixel intensity relationships between images dominate the PCA co-variance matrix; in breath-hold DCE-MRI, contrast enhancement dominates since there are many intensity relationships between images. The PCA separates long-term (i.e. over the entire dataset) temporal enhancement trends into early principal components and short-term (i.e. over a few images) temporal motion trends, especially those in which organ position is not correlated between time-points, into later principle components. Motion that is periodic may be expected to produce more image-to-image relationships, since the images would be aligned in groups, depending on the number of cycles, influencing the co-variance matrix.

$$
\mathbf{A}_{i j}(t) \approx \sum_{m=1}^{\eta \leq M}\left(\mathbf{U}_{m} \cdot \mathbf{A}_{i j}(t)\right) \mathbf{U}_{m}
$$


The registration method is shown in the algorithm in 4 where the result at the next iteration, $n+1$, is given by the registration $(\mapsto)$ of the best registered data from the previous step $n$, registered to artificial images generated from a temporal principal components analysis of the registered data from the previous step $n$, rebuilt using $n$ principal components (hence including the same number of principal components as the iteration number). Registration of source images to artificial target images uses a fluid registration algorithm [9] with a crosscorrelation cost function, appropriate for the images being registered.

$$
\mathbf{A}_{i j}(t)^{n+1}=\mathbf{A}_{i j}^{n}(t) \mapsto \sum_{m=1}^{n<M}\left(\mathbf{U}_{m}^{n} \cdot \mathbf{A}_{i j}^{n}(t)\right) \mathbf{U}_{m}^{n}
$$

\section{Results}

DCE-MRI datasets are generated for a range of motions and enhancements. Two types of motion are considered, cyclic motion due to breathing-like motion and breath-hold depth inconsistency in which liver position is determined by a Gaussian distribution around zero displacement (this simulation is consistent with the data presented in 4). Different motion levels 1-4 (level 4 corresponds to a maximum of 20 pixels $(35 \mathrm{~mm})$ displacement) are considered. Different levels of enhancement are included, with scaling levels 0-3 (with level 3 corresponding to a maximum increase of $50 \%$ pixel intensity). Two registration types are compared, the first is a direct (fluid) registration of each image in the simulated dataset to the first image in the dataset, the second is the PPCR algorithm.

We analyse: residual motion in the deformation fields; found by taking the gold-standard deforming transformation and adding to it the correcting registration displacement field; tumour volume, both to assess the volume preserving constraint and to observe any additional tumour volume changes due to the registration process; the joint image histograms of the unregistered and the first PPCR target images to analyse the contribution of dispersion from contrast enhancement and motion.

Figures 1a-d show the residual deformation after registration, demonstrating PPCR registration errors increasing with the amplitude of periodic motion. The shape of the unregistered deformation is a consequence of elliptical motion, taking different paths during inhale and exhale. Registration by PPCR for large periodic motion separates the final images into clusters at two locations. This is due to the periodic motion influencing early principal components, generating target images that are ill-defined (Figure 20), containing a spread of image positions. Images in different phases of breathing are successively driven toward separate locations during subsequent iterations, since these are reinforced in the second calculation of the PCA, producing clusters of well-registered images. If the final PPCR clusters are distinct, we can manually correct this effect by finding the transform between clusters, thus bringing the PPCR result to an equivalent correction to direct registration. Since images within the separate locations are well registered to one another, we require only a single deformation 

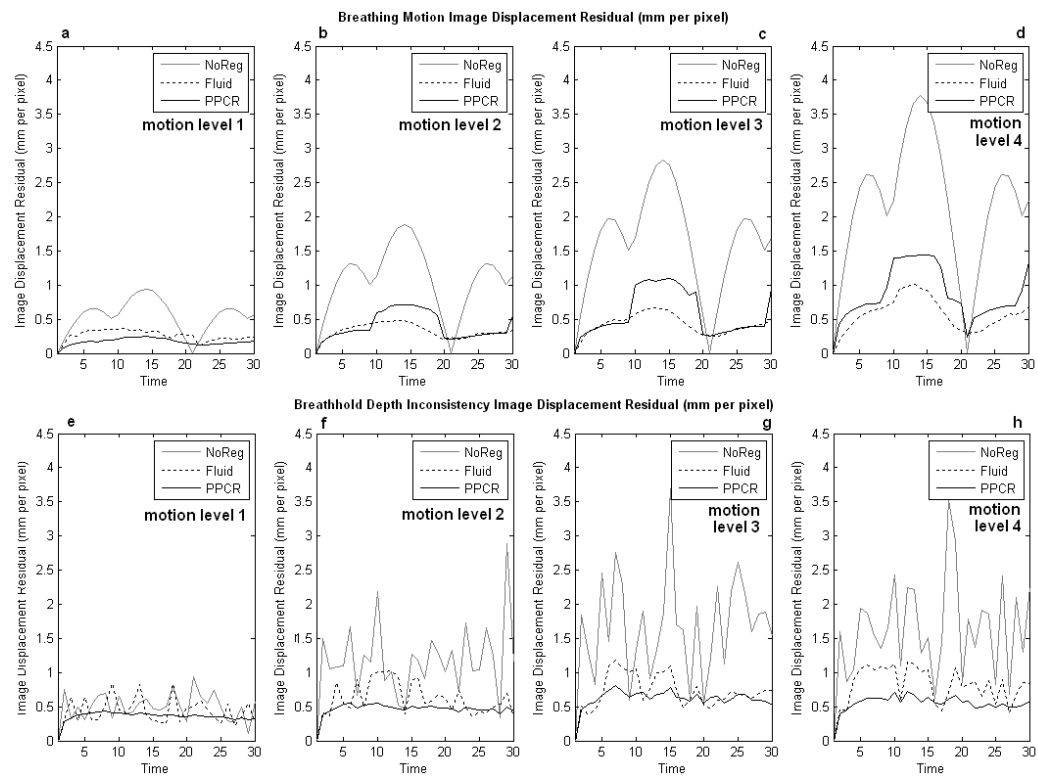

Fig. 1. Graphs of absolute image residual displacement with time (image number) for varying motion (levels $1-4$ corresponding to $9 \mathrm{~mm}, 18 \mathrm{~mm}, 26 \mathrm{~mm}, 35 \mathrm{~mm}$ maximum displacements. a-d Cyclic breathing motion for contrast-enhancement level 3 showing separation of final registration position between two locations see text. $\mathbf{e}-\mathbf{h}$ Breathhold depth inconsistency for contrast-enhancement level 3 showing artefacts under increasing enhancement for direct fluid registration.

between clusters. The transformation is determined by the registration between images either side of the first jump between clusters and applied to all images within the cluster. This correction should be implemented in the first iteration of the PPCR method; inspecting the inter-image residual deformation allows clusters to be determined and then removed as above.

With no enhancement, both fluid and PPCR demonstrate successful registration, illustrated by a reduction in the residual motion. With increasing enhancement, fluid registration begins to mis-register enhancing regions, particularly visible in the images as distortions to the rigid tumours. This effect is shown as a failure to reduce the residual deformation level. Figures 1 $\mathrm{k}$-h demonstrate the difference in registration success for fixed breath-hold consistency level and varying contrast enhancement. For level 3 enhancement, the fluid registration is actively mis-registering a large proportion of the images. The eventual success of the PPCR method can be seen in the first target image shown in Figure 2. in which features are given a well-defined average position.

Figure 3 a plots fluctuations in tumour size. It is clear that the tumour volumepreserving modification is not entirely successful, compressing the tumour up to $1 \%$ with increasing motion levels. The failure of the modification is less important when considering volume change due to the direct fluid registration. Volume 


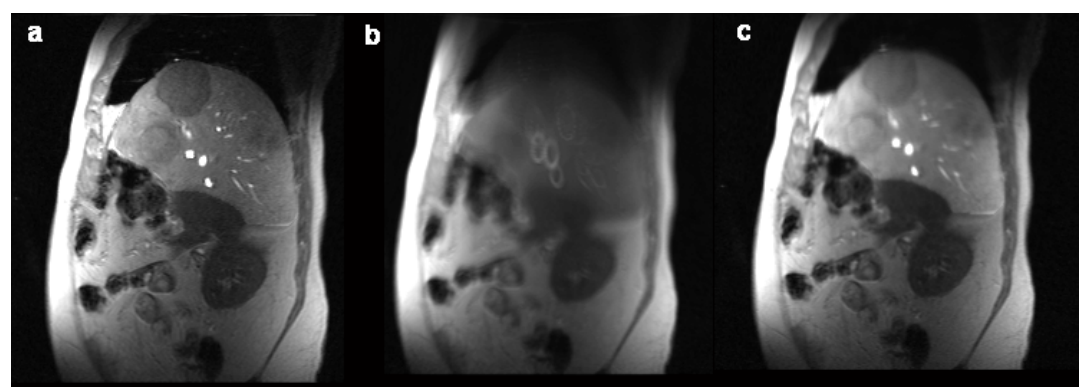

Fig. 2. a) Source image from dataset with motion level 4 (up to $35 \mathrm{~mm}$ displacement) and enhancement level 3. b) PPCR generated target image from first iteration for model with cyclic breathing motion for comparison with c) PPCR generated target image from first iteration for model with breath-hold depth inconsistency. The illdefined nature of $\mathbf{b}$ results in separation of final registration position see text.

change is visually correlated with the strength of contrast enhancement and volume changes of up to $7 \%$ are observed, visible in the actual images. Tumour volume change is never more than 1\% using the PPCR method. Since tumours are the very objects we are likely to be interested in, the success of registration in this region is crucial.

Joint image histograms between pre- and post- enhancement images contain dispersion from both motion and contrast enhancement processes. Histogram dispersion is a result of one-to-one pixel intensity relationships becoming oneto-many due to spatially dependent intensity variations. Crudely, this is seen as a second arm in Figure 4 a for breath-hold depth inconsistency of level 3 with enhancement level 3. Information based cost-functions aim to minimise this dispersion but do not distinguish between the two sources, this is also true of statistical cost functions such as cross-correlation. For PPCR generated target images, the contrast enhancement level is more closely matched since gross intensity changes are encoded in the early principal components, therefore dispersion due to contrast enhancement is removed and the remaining dispersion is more strongly associated with motion. This can be seen in Figure 3 ; as an enhancement dependent reduction in the total dataset joint entropy between source images and PPCR generated target images. However, if the PPCR target images do not well-match the source images, the dispersion will not decrease and theoretically could increase (Figure $2 \mathrm{~b}$ is an example target image from the dataset for motion level 4 enhancement level 3 with corresponding point in for motion level 4, enhancement level 3 in Figure 3b: there is no overall adjustment to joint entropy). Blurring of the joint histogram is always likely to occur (there is slight additional dispersion in $4 \mathrm{~b}$ ). This is because PPCR generates target images in early iterations that contain averaged representations of enhancement, but if the reduction in dispersion due to contrast enhancement is greater than this additional blurring, registration by PPCR may proceed. 

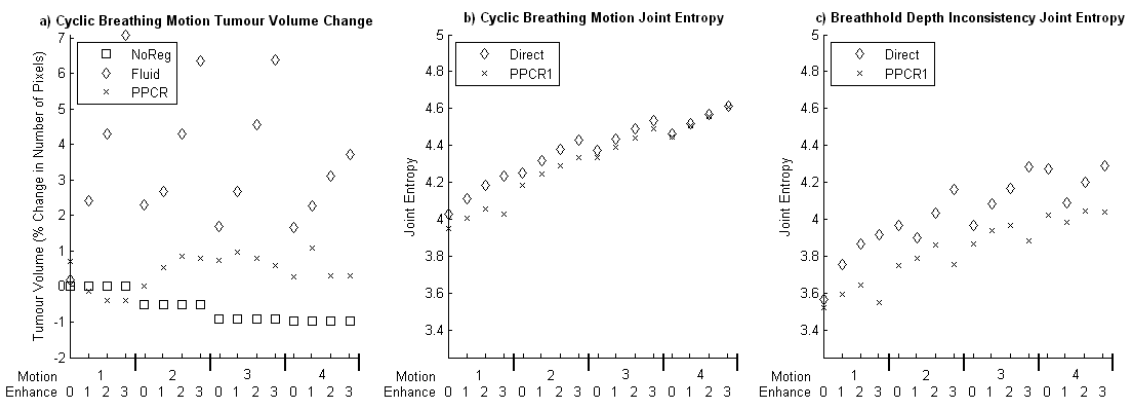

Fig. 3. Graphs plotting statistics for variations in a) tumour volume change for breathing-motion extent and enhancement level, note the trend for decreasing tumour size in the gold standard. b) average joint entropy of target and source images per simulation for direct registration (to pre-contrast image) and to first set of target images generated by PPCR for breathing motion. $\mathbf{c}$ for breath-hold depth inconsistency. Note increasing average joint entropy with motion and enhancement.

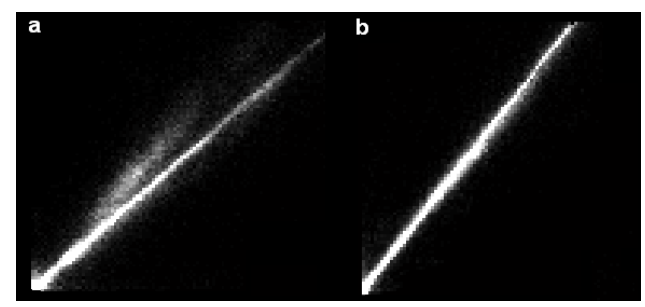

Fig. 4. Joint Image Histograms for spatial displacement due to breath-hold depth inconsistency (level 3) between a) pre and post enhancement level 3 images, b) post enhancement image and corresponding PPCR generated post enhancement image (first PPCR iteration)

\section{Discussion}

We have shown the applicability of two image registration methods applied to synthetic DCE-MRI generated with different levels of motion and enhancement. Under cyclic breathing motion, PPCR finds it difficult to generate a representative set of target images (Figure 1 $1 \mathrm{a}-\mathrm{d}$ ), but this type of motion produces a predictable result, requiring a modification or diagnosis that should be included in the algorithm. The PPCR method is able to preserve the volume of enhancing regions unlike direct registration which fails under increasing contrast enhancement. This is a failure of the cost-function; using information based cost-functions, joint histogram dispersion from artefactual feature misalignment and from valid contrast enhancement is penalised. By encapsulating intensity variations in early principal components, PPCR generates enhancement matched target images, reducing dispersion in the joint histogram due to contrast 
enhancement, which is why the method works. This allows registration to proceed, but only in cases where target images are well-matched to their source images, which is not the case under cyclic breathing motion. In this case, there is an increase in dispersion due to poor target matching that offsets the reduction due to contrast matching and PPCR will break down. Future work will develop the breathing-model to allow for more realistic unpredictability in breathingdepth and phase, improve the enhancement model to make it organ specific, extend the work fully to three dimensions.

\section{References}

1. Cuenod, C.A., Fournier, L., Balvay, D., Guinebretière, J.M.: Tumor angiogenesis: pathophysiology and implications for contrast-enhanced MRI and CT assessment. Abdom Imaging 31, 188-193 (2006)

2. Tofts, P.S., Brix, G., Buckley, D.L., Evelhoch, J.L., Henderson, E., Knopp, M.V., Larsson, H.B., Lee, T.Y., Mayr, N.A., Parker, G.J., Port, R.E., Taylor, J., Weisskoff, R.M.: Estimating kinetic parameters from dynamic contrast-enhanced T(1)weighted MRI of a diffusable tracer: standardized quantities and symbols. J. Magn. Reson. Imaging 10, 223-232 (1999)

3. Buonaccorsi, G.A., Roberts, C., Cheung, S., Watson, Y., O'Connor, J.P.B., Davies, K., Jackson, A., Jayson, G.C., Parker, G.J.M.: Comparison of the performance of tracer kinetic model-driven registration for dynamic contrast enhanced MRI using different models of contrast enhancement. Acad. Radiol. 13, 1112-1123 (2006)

4. Melbourne, A., Atkinson, D., White, M.J., Collins, D., Leach, M., Hawkes, D.: Registration of dynamic contrast-enhanced mri using a progressive principal component registration (ppcr). Phys. Med. Biol. 52, 5147-5156 (2007)

5. Blackall, J.M., Ahmad, S., Miquel, M.E., McClelland, J.R., Landau, D.B., Hawkes, D.J.: Mri-based measurements of respiratory motion variability and assessment of imaging strategies for radiotherapy planning. Phys. Med. Biol. 51, 4147-4169 (2006)

6. Rohlfing, T., Maurer, C.R., O'Dell, W.G., Zhong, J.: Modeling liver motion and deformation during the respiratory cycle using intensity-based nonrigid registration of gated MR images. Med. Phys. 31, 427-432 (2004)

7. Tofts, P.S., Kermode, A.G.: Measurement of the blood-brain barrier permeability and leakage space using dynamic MR imaging. 1. Fundamental concepts. Magn. Reson. Med. 17, 357-367 (1991)

8. Sarvazyan, A., Goukassian, D., Maevsky, E., Oranskaja, G.: Elastic imaging as a new modality of medical imaging for cancer detection. In: International Workshop on Interaction of Ultrasound with Biological Media, pp. 69-81 (1994)

9. Crum, W.R., Tanner, C., Hawkes, D.J.: Anisotropic multi-scale fluid registration: evaluation in magnetic resonance breast imaging. Phys. Med. Biol. 50, 5153-5174 (2005) 